Schlömerkemper, Jörg

\title{
"Nomen est omen" - oder geht es auch ohne? Was kann/Was soll der Name einer Person bei einem Preis bedeuten? Anmerkungen nach dem Streit um die Benennung des Forschungspreises der DGfE nach Heinrich Roth
}

Erziehungswissenschaft 25 (2014) 49, S. 47-48

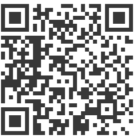

Quellenangabe/ Reference:

Schlömerkemper, Jörg: "Nomen est omen" - oder geht es auch ohne? Was kann/Was soll der Name einer Person bei einem Preis bedeuten? Anmerkungen nach dem Streit um die Benennung des Forschungspreises der DGfE nach Heinrich Roth - In: Erziehungswissenschaft 25 (2014) 49, S. 47-48 URN: urn:nbn:de:0111-pedocs-100802 - DOI: 10.25656/01:10080

https://nbn-resolving.org/urn:nbn:de:0111-pedocs-100802

https://doi.org/10.25656/01:10080

in Kooperation mit / in cooperation with:

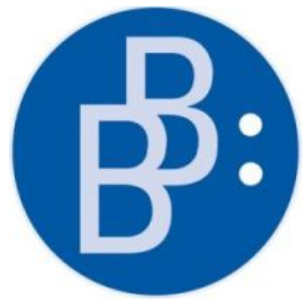

https://www.budrich.de

\section{Nutzungsbedingungen}

Gewährt wird ein nicht exklusives, nicht übertragbares, persönliches und beschränktes Recht auf Nutzung dieses Dokuments. Dieses Dokument is ausschließlich für den persönlichen, nicht-kommerziellen Gebrauch bestimmt. Die Nutzung stellt keine Übertragung des Eigentumsrechts an diesem Dokument dar und gilt vorbehaltlich der folgenden Einschränkungen: Auf sämtlichen Kopien dieses Dokuments müssen alle Urheberrechtshinweise und sonstigen Hinweise auf gesetzlichen Schutz beibehalten werden. Sie dürfen dieses Dokument nicht in irgendeiner Weise abändern, noch dürfen Sie dieses Dokument für öffentliche oder kommerzielle Zwecke vervielfältigen, öffentlich ausstellen, aufführen, vertreiben oder anderweitig nutzen.

Mit der Verwendung dieses Dokuments erkennen Sie die Nutzungsbedingungen an.

\section{Terms of use}

We grant a non-exclusive, non-transferable, individual and limited right to using this document.

This document is solely intended for your personal, non-commercial use. Use of this document does not include any transfer of property rights and it is conditional to the following limitations: All of the copies of this documents must retain all copyright information and other information regarding legal protection. You are not allowed to alter this document in any way, to copy it for public or commercial purposes, to exhibit the document in public, to perform, distribute or otherwise use the document in public.

By using this particular document, you accept the above-stated conditions of use.

\section{Kontakt / Contact:}

peDOCS

DIPF | Leibniz-Institut für Bildungsforschung und Bildungsinformation Informationszentrum (IZ) Bildung

E-Mail: pedocs@dipf.de

Internet: www.pedocs.de

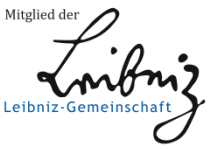




\section{Erziehungswissenschaft}

Mitteilungen der Deutschen Gesellschaft

für Erziehungswissenschaft (DGfE)

Heft 49

25. Jahrgang 2014

ISSN 0938-5363

Verlag Barbara Budrich 


\section{INHALTSVERZEICHNIS}

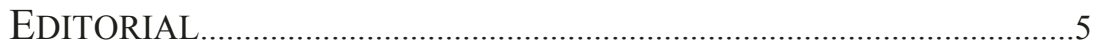

BEITRÄGE ZUM THEMA ,ERINNERUNGSKULTUREN PREISE, NAMEN UND DISZIPLINPOLITIK“

Stellungnahme des Vorstandes zur Rücknahme des Namens für den

Forschungspreis vom 13. Januar 2014

Andreas Hoffmann-Ocon

Heinrich Roth und der Preis der Forschung - Historisierungen,

Verundeutlichungen und erziehungswissenschaftliche

Erinnerungsgeschichten

Benjamin Hasselhorn

Erinnerung im Streit. Zum Umgang der deutschen

Geschichtswissenschaft mit ihrer eigenen Vergangenheit

Eva Matthes \& Carola Groppe

Historische Bildungsforschung und Erinnerungskultur. Eine

Stellungnahme der Sektion Historische Bildungsforschung.

Daniel Tröhler

Wissenschaftliche Ehrungen oder der doppelte Gestus der

Auszeichnung. Bemerkungen zur fallengelassenen Idee eines Heinrich

Roth-Forschungspreises der DGfE

Jörg Schlömerkemper

„Nomen est omen“ - oder geht es auch ohne? Was kann/Was soll der

Name einer Person bei einem Preis bedeuten? Anmerkungen nach dem

Streit um die Benennung des Forschungspreises der DGfE nach

Heinrich Roth

BEITRÄGE

Adolf Kell

Grenzgänge, Traditionen und Zukünfte in der Deutschen Gesellschaft für Erziehungswissenschaft. Kongresse zur Reflexion - auch für die Sektion Berufs- und Wirtschaftspädagogik? 


\section{MitTEILUNGEN DES VORSTANDS}

Protokoll der Mitgliederversammlung der Deutschen Gesellschaft für

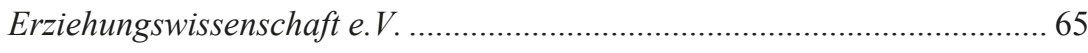

Ergebnis der Wahl zum Vorsitz und Vorstand der DGfE............................ 71

Liste der unbekannt verzogenen Mitglieder ............................................... 72

BERICHTE AUS DEN SEKTIONEN

Sektion 1 - Historische Bildungsforschung ............................................... 75

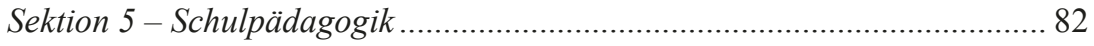

Sektion 8 - Sozialpädagogik und Pädagogik der frühen Kindheit................ 85

Sektion 9 - Erwachsenenbildung .............................................................. 91

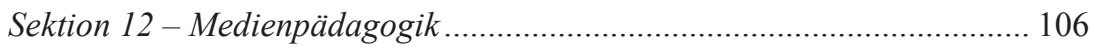

Sektion 13 - Differentielle Erziehungs- und Bildungsforschung ................ 108

NOTIZEN

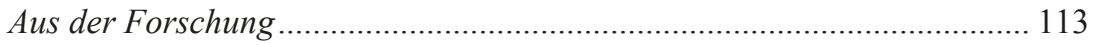

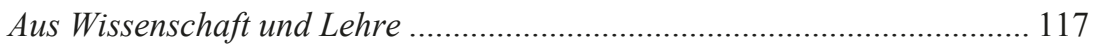

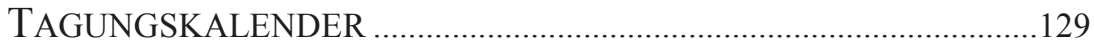




\section{„Nomen est omen“ - oder geht es auch ohne?}

\section{Was kann/Was soll der Name einer Person bei einem Preis bedeuten? Anmerkungen nach dem Streit um die Benennung des Forschungspreises der DGfE nach Heinrich Roth}

\section{Jörg Schlömerkemper}

Man kann mit zweifellos guten oder zumindest gut gemeinten Argumenten darüber streiten, ob es sinnvoll ist, einen ausgelobten Preis mit dem Namen einer Persönlichkeit zu verbinden. Prinzipiell ist dies meines Erachtens nicht $\mathrm{zu}$ entscheiden. Es kommt vielmehr darauf an, was man überhaupt mit der Vergabe eines Preises bewirken will. Vor allem zwei Perspektiven scheinen dabei bedeutsam zu sein:

In erster Linie wird es darum gehen, eine herausragende Leistung für die jeweilige Wissenschaft zu würdigen und zu signalisieren, dass man für die zukünftige Entwicklung eine Fortführung dessen für wünschenswert hält, wofür die Preisträgerin bzw. der Preisträger gelobt wird. Das kann tendenziell sogar auf einen Perspektivenwechsel oder eine neue Schwerpunktsetzung zielen. Dann ist es vielleicht gar nicht möglich, eine bestimmte Person, die in der Regel bereits verstorben sein dürfte, mit einem solchen Wunsch zu verbinden. Zum anderen kann man aber gerade gegen vermeintlich ,modische“ Entwicklungen ein Signal setzen wollen, indem man ausdrücklich an Traditionen anknüpft und dies mit der Hervorhebung einer bestimmten Persönlichkeit zum Ausdruck bringen will. Solche Reminiszenzen kann man natürlich als ,traditionalistisch' einschätzen und der Meinung sein, dass dies nicht mehr in unsere Zeit passt. Man solle doch lieber sachlich nüchtern sagen, welche Themen, Programme oder Praxen man für besonders wichtig hält und fördern möchte.

Man muss also wissen, was man will, und entsprechend entscheiden. Plädieren würde ich dafür, dass man versucht, das eine mit dem anderen zu verbinden. Eine Sentenz Odo Marquards erinnert an die Notwendigkeit einer doppelten Blickrichtung: „Zukunft braucht Herkunft“ (Marquard 2003). Auch eine wissenschaftliche Disziplin sollte die Erinnerung an ihre historische Entwicklung pflegen und im Bewusstsein halten, welche Konzepte umstritten waren, welche sich durchgesetzt haben bzw. durchgesetzt worden sind, aber auch welche nicht im Mainstream bewahrt werden.

Dabei kann man natürlich über die Bedeutung der verschiedenen Forschungsparadigmen etc. trefflich streiten und es wird immer wieder unterschiedliche Einschätzungen oder gar kontroverse Debatten geben. Aber gerade dies ist eine Voraussetzung dafür, dass eine wissenschaftliche Disziplin lebendig bleibt, und es dürfte ihr zur Ehre gereichen, wenn es gelingt, solche 
Diskussionen differenziert und mit dem gebührenden Respekt für gegenteilige Positionen zu führen. In einer Disziplin, die sich u.a. mit dem Verhältnis der Generationen und mit pädagogischer Interaktion beschäftigt, dürfte dies in besonderer Weise wünschenswert sein.

Für eine deutsche wissenschaftliche Gesellschaft ist der Blick in die Herkunft sogleich mit Problemen verbunden, wenn eine Persönlichkeit zur Benennung eines Preises in Erwägung gezogen wird, die bereits vor 1945 wissenschaftlich gearbeitet hat. Die Lebensleistungen vieler solcher Persönlichkeiten, die aus ganz verschiedenen Gründen in Deutschland geblieben sind, können bei historischen Recherchen in ein Zwielicht geraten und mit eindeutig anspruchsvollen Maßstäben geleugnet werden. Entsprechende Äußerungen und ggf. Verhaltensweisen sollten allerdings im Kontext jener Zeit bewertet werden, in der sie getätigt worden sind. Verurteilungen aus der Perspektive absoluter moralischer Ansprüche scheinen mir wenig geeignet zu sein, die Verstrickungen, in die jemand geraten ist oder in die jemand sich möglicherweise fahrlässig selbst begeben hat, genauer zu analysieren und mit der Perspektive zu verstehen, dass solche Entwicklungen sich nicht wiederholen können. Letztendlich ist es entscheidend, welche Lebensleistung eine Persönlichkeit insgesamt vollbracht hat und ob sich darin ausdrücken lässt, was die Verleihung eines Preises bedeuten soll.

In diesem Sinne hatte ich es als angemessen und erfreulich empfunden, dass die Deutsche Gesellschaft für Erziehungswissenschaft einen Forschungspreis mit den Namen Heinrich Roth verbinden wollte. Damit wäre zweierlei zum Ausdruck gebracht worden: Zum einen wäre deutlich geworden, dass empirischer Forschung - so wie Heinrich Roth die von ihm geforderte "Wendung“ gemeint hatte - in eine hermeneutische bzw. philosophische Reflexion eingebunden sein muss. Und zum anderen wäre deutlich geworden, dass erziehungswissenschaftliche Forschung verpflichtet ist, sich auf gesellschaftliche Entwicklungen und individuelle Entfaltungsmöglichkeiten $\mathrm{zu}$ beziehen und darauf nach Möglichkeit einzuwirken.

Unter diesen beiden Aspekten hätte der Name Heinrich Roth dem Forschungspreis Bedeutung geben können. Dass der Preis an Eckhard Klieme verliehen wurde, lässt allerdings erkennen, dass diese beiden Intentionen bei der Entscheidung über den Preisträger offenbar eine Rolle gespielt haben.

Jörg Schlömerkemper, Prof. Dr. i.R., war bis 2008 Hochschullehrer an der Goethe-Universität Frankfurt am Main im Fachbereich Erziehungswissenschaften.

\section{Literatur}

Marquard, Odo (2003): Zukunft braucht Herkunft. Stuttgart: Reclam. 